\title{
THOMAS SARGENT FACE A ROBERT LUCAS : UNE AUTRE AMBITION POUR LA NOUVELLE ECONOMIE ClassiQue
}

\author{
Aurélien Goutsmedt ${ }^{1}$
}

\begin{abstract}
RESUME
L'article montre que la vision de la macroéconomie de Sargent contraste d'avec celle de Lucas. Pour Lucas, les hypothèses d'un modèle sont " a-réalistes », le modèle ne vise pas à représenter la réalité. Il est un outil de simulation qui doit permettre de simuler différentes politiques économiques. L'idéal « lucassien » est celui d'un macroéconomiste qui a donc vocation à devenir un ingénieur chargé de fournir un « logiciel de politiques économiques » aux autorités publiques, logiciel qu'il manipule afin d'aiguiller les choix de politiques sur une base scientifique. Sargent, quant à lui, considère que pour suppléer le paradigme keynésien, la nouvelle économie classique doit être capable de remplir les mêmes tâches, et l'une de ces tâches est de conseiller le pouvoir en lui fournissant une grille de lecture des phénomènes économiques et des outils intuitifs pour débattre des politiques économiques à mettre en place. Sargent cherche à appliquer ce qu'il nomme la théorie des anticipations rationnelles à un ensemble de cas concrets (stabilisation Poincaré, hyperinflation allemande, politique de Thatcher et Reagan) pour montrer la pertinence de ce cadre d'analyse pour penser les problèmes économiques contemporains.
\end{abstract}

Mots Clés : Histoire de la macroéconomie ; Modèles macroéconomiques ; Nouvelle Economie Classique ; Stagflation

Codes JEL : B22 ; E52 ; N12.

\section{INTRODUCTION}

Au milieu des années 1980, la majorité des macroéconomistes s'accorde pour dire que la théorie macroéconomique ne ressemble sensiblement plus à ce qu'elle pouvait être deux décennies plus tôt. Ce qu'on appelait alors la « révolution des anticipations rationnelles » (Begg, 1982) avait profondément secoué les fondements de la discipline. Ces transformations sont portées dans les années 1970 par un petit groupe d'économistes qu'on appelle rapidement « nouveaux classiques $»^{2}$. Ils revendiquent pour la discipline

\footnotetext{
${ }^{1}$ Université Paris 1, Panthéon-Sorbonne. Centre d'économie de la Sorbonne. Aurelien.goutsmedt@univparis1.fr. Je tiens à remercier Annie L. Cot, Michel De Vroey, Jérôme Lallement, Jacques Le Cacheux, Matthieu Renault, et Francesco Sergi pour leur lecture d'une version antérieure de cet article, ainsi que les trois rapporteurs anonymes dont les conseils et remarques ont été fort utiles. Les erreurs qui demeurent me sont bien entendu imputables.

${ }^{2}$ Le terme est désormais répandu pour définir le groupe d'économistes autour de Lucas et Sargent, dans les années 1970 et au début des années 1980. Le terme de «modèles nouveaux classiques » apparaît sous
} 
de nouveaux objets, ainsi qu'une nouvelle façon de construire des modèles, une nouvelle articulation entre modèle et théorie, et de nouveaux critères de test pour éprouver la solidité et la validité d'un modèle. D'après l'un de ces économistes, il ne s'agit plus « simplement de proposer de nouvelles réponses à de vieilles questions, mais d'offrir une nouvelle vue des questions qu'il est utile de poser » (Sargent, 2013, xxi) ${ }^{3}$.

Un personnage se détache sur la scène académique : Robert Lucas, acclamé en tant que « maître de la méthodologie » (Edward Prescott, dans son interview avec Snowdon et Vane, 2005, 351) et considéré par nombre de macroéconomistes comme l'un des architectes de la macroéconomie moderne (Hall, 1996, Woodford, 2003). Paradoxalement, on ne trouve chez lui aucun article à proprement parler méthodologique, excepté peut-être son "Methods and Problems in Business Cycle Theory » (Lucas, 1980a). Mais il est possible de reconstruire sa position à partir d'un grand nombre d'éléments égrainés dans ses contributions à la théorie macroéconomique des années 1970 (voir Vercelli, 1991, De Vroey, 2015). Se détache alors la défense d'une forme particulière d'instrumentalisme méthodologique. Pour ne pas trop anticiper le propos qui va suivre, on pourrait caricaturer la position de Lucas de la sorte : en tant qu'il représente une économie artificielle abstraite, un modèle est nécessairement non-réaliste. Le critère de validité d'un modèle est à chercher dans sa capacité à reproduire, à imiter, des faits économiques quantitatifs et dans sa capacité à répondre à des questions de politique économique. Dès lors, il n'est pas approprié pour Lucas de questionner le réalisme des hypothèses utilisées dans le modèle. L'économie modélisée doit être clairement distinguée des systèmes économiques du monde réel. Le but principal de la macroéconomie, pour Lucas, devient l'évaluation de politiques économiques. La question de la capacité des modèles à faciliter l'interprétation et l'explication des phénomènes macroéconomiques est reléguée au second plan (voire disparaît parfois totalement).

Par son statut, Lucas est érigé comme figure de proue de la nouvelle économie classique (NEC) et la méthodologie qu'il esquisse au travers de ses contributions des années 1970 tend à être perçue comme représentative des prétentions épistémologiques des nouveaux classiques dans leur ensemble. L'article se propose de souligner le caractère réducteur de cette tentation à la généralisation et à l'extension de la conception de Lucas, en s'intéressant aux propos méthodologiques de l'un de ses principaux coauteurs, Thomas Sargent, qui apporte une dimension supplémentaire au projet de la NEC au début des années 1980.

Pour dresser cette comparaison avec Lucas, je m'appuierai sur certaines contributions de Sargent publiées dans la première moitié des années 1980, et regroupées dans un livre, Rational Expectations and Inflation (Sargent, 2013) ${ }^{4}$. Les différentes préfaces de ce

la plume de (Tobin, 1980) et l'on parle également de «nouvelle macroéconomie classique » (Buiter, 1980 ; Hoover, 1988 ; Klamer, 1984) ou de «nouvelle économie classique » (Stein, 1981).

3 Je traduis de l'anglais une partie des citations.

${ }^{4}$ Ce livre a connu trois éditions, dont la première (celle qui nous intéresse), date de 1985. Quatre articles, reproduits dans cet ordre dans l'ouvrage, permettent d'alimenter mon propos : "Rational Expectations and the Reconstruction of Macroeconomics » (Sargent, 1980), « Reaganomics and Credibility » (1985), 
livre, réédité deux fois, sont révélatrices de l'objectif global de Sargent et de sa conception du travail du macroéconomiste. Il s'affirme comme le défenseur d'une vision plus réaliste que Lucas des modèles de la NEC, au sens de réalisme scientifique : dans une perspective réaliste, le contenu d'un modèle se réfère au monde réel et vise à décrire par approximation certaines particularités de ce monde (Mäki, 2012, 4). Quant au terme d'instrumentalisme qui nous permet de caractériser Lucas, il renvoie à la vision d'un modèle comme un instrument, un outil, pour atteindre un certain objectif (ici, l'évaluation de politiques économiques). Et toute la construction du modèle est soumise à cet impératif, la question de la capacité du modèle à se référer au monde réel et à le décrire étant ainsi reléguée à l'arrière-plan (voir Mäki, 2009a).

Cet article défend l'idée qu'à travers l'étude des propos méthodologiques de Lucas et Sargent, dans les années 1970 et au début des années 1980, on voit apparaître deux stratégies différentes sur l'usage des modèles macroéconomiques à cette époque. Tout en s'appuyant sur le même arrière-plan analytique que Lucas (hypothèse d'apurement permanent des marchés, microfondements des modèles à partir de problèmes d'optimisation intertemporelle et utilisation des anticipations rationnelles; voir Hoover, 1988, 12-14), et tout en adhérant à certains moments à son approche méthodologique, Sargent s'évertue, au début des années 1980, à défendre une utilisation des modèles de la NEC plus étendue et en prise avec des problèmes macroéconomiques concrets, à travers plusieurs études historiques et contemporaines. L'objectif à l'époque est de favoriser l'ascension de la NEC au sein du champ de la macroéconomie en rendant ses travaux plus séduisants.

Alors même que l'on pourrait arguer d'une contradiction apparente entre Lucas et Sargent sur la question méthodologique, ces deux stratégies s'avèrent en réalité complémentaires pour s'imposer dans la discipline. Face à la persistance de l'inflation, au ralentissement de la croissance et à la montée du chômage, la macroéconomie est un lieu de débats et d'affrontements intenses à la fin des années 1970 et au début des années 1980. Lucas cherche à profiter de la déstabilisation du consensus keynésien des années 1960 pour redéfinir les règles de la pratique macroéconomique. Il s'efforce de réaffirmer l'autonomie de la discipline, face aux pressions extérieures et à la conjoncture présente, par la défense d'une forme de pureté méthodologique qui exige de refonder la macroéconomie sur les principes de la théorie de l'équilibre général, telle que formulée par Arrow et Debreu (1954). Lucas s'efforce d'opérer alors un cloisonnement rigide entre l'économie artificielle modélisée par le macroéconomiste et les " économies »du monde réel. Ainsi, on ne trouvera pas dans ses travaux d'explication directe et explicite de la stagflation, ni de proposition de remède (ce qui n'empêche pas d'extrapoler à partir des modèles qu'il produit). Or, alors même que la remise en cause du consensus keynésien, dans les années 1970, est en partie liée au contexte économique et politique, et aux erreurs de prédiction des modèles macroéconométriques structurels, les

«The Ends of four big Inflations » (1982) et « Stopping Moderate Inflations: the Methods of Poincaré and Thatcher » (1983). 
nouveaux classiques ne semblent pas offrir de discours de substitution sur la situation économique présente 5 .

Cependant, au début des années 1980, Sargent cherche à " opérationnaliser » les modèles de la NEC pour en faire un outil plus flexible pour justifier des prises de position dans le champ de la politique économique. Ces modèles, reposant sur l'optimisation intertemporelle et les anticipations rationnelles, ont alors quelque chose à nous dire sur la stagflation qui se déroule aux Etats-Unis, et sur les moyens d'en sortir. Or, notre hypothèse sous-jacente est que, au vu de l'importance cruciale à l'époque du problème de l'inflation, la NEC n'aurait pu connaître un tel succès sans aussi se montrer capable d'être partie prenante des débats contemporains. Ou, pour le dire de manière plus provocatrice, il faut un Sargent (celui qui nous intéresse dans l'article) pour que Lucas puisse être Lucas ${ }^{6}$. La volonté de Sargent d'opérationnaliser et d'utiliser de manière concrète les anticipations rationnelles complète ainsi la stratégie de distanciation de Lucas.

Pour appuyer ce propos, une première section s'attachera à exposer la méthodologie de Lucas. Cette dernière est bien connue et a déjà fait l'objet de plusieurs reconstructions complètes ou partielles (Boumans, 1997, De Vroey, 2009; 2015, Vercelli, 1991). L'objectif sera de cibler les particularités de cette méthodologie qui révèlent le contraste existant avec Sargent (Section 1). Le contraste deviendra d'autant plus clair avec l'exposition de l'ambition proclamée par Sargent de faire de ce qu'il appelle la « théorie des anticipations rationnelles » un outil de pensée à visée heuristique et pratique, à la manière du modèle IS-LM (Section 2). La dernière section sera l'occasion de voir cette théorie des anticipations rationnelles en action. Sargent s'attache à réinterpréter un certain nombre d'événements économiques courants et historiques à l'aune de cette grille de lecture, ce qui lui permet de prendre part aux débats sur l'efficacité potentielle d'une politique de désinflation et sur la manière de la mettre en pratique (Section 3).

\section{L'INSTRUMENTALISME METHODOLOGIQUE DE ROBERT LUCAS}

Cette section porte son attention sur les principes méthodologiques esquissés par Lucas tout au long de ses contributions regroupées dans Studies in Business-Cycle Theory. Elle s'appuie également sur les analyses qu'en proposent De Vroey (2015, chapitres 10 et

\footnotetext{
${ }^{5}$ L'idée n'est pas d'affirmer que les modèles de la NEC n'ont inspiré aucune explication de la crise, ou aucune proposition de politique économique, durant les années 1970. On constate d'ailleurs que les travaux des nouveaux classiques commencent à trouver un certain écho dans la presse (Willes, 1978). Cependant, les nouveaux classiques (ou du moins Lucas, plus particulièrement) semblent encore absents à ce moment des débats extra-académiques sur la conjoncture et la politique économique et réticents à défendre une grille d'analyse de la situation et des propositions explicites. Paradoxalement, c'est chez les keynésiens que l'on trouve les principales analyses fouillées et systématiques du phénomène de stagflation (Blinder, 1979, Solow, 1980, Bruno, 1985).

${ }^{6}$ Il faut un Sargent, et sans doute bien d'autres choses encore, tels que des relais dans la presse ou dans les think tanks qui se développent à l'époque (Cockett, 1995, Edwards, 2013, Smith, 1993), ou bien un groupe de recherche actif à la Federal Reserve Bank de Minneapolis (Miller, 1994, McGregor, 2013) qui permet de se faire entendre au sein du Federal Open Market Committee.
} 
11) et Sergi (2017, chapitre 1). Ces analyses réalisent une reconstruction rationnelle des différents éléments méthodologiques égrainés par Lucas dans différents textes. A travers ces textes, on voit apparaître une vision idéalisée et fantasmée de ce que devrait être, pour lui, la macroéconomie. Idéalisée, car Lucas n'ignore pas le fait que ce qu'il préconise ne va pas sans contradictions et complications (voir infra et Sergi, 2017, chapitre 1). Fantasmée, car Lucas revendique la généralisation de pratiques qu'il est pour le moment lui-même bien incapable de mettre en place ${ }^{7}$. Mais c'est cette vision qui nous intéresse, car elle témoigne de la volonté d'engager plus avant un processus d'autonomisation de la macroéconomie par rapport aux pressions extérieures (c'est-àdire par rapport à la demande sociale et politique présente), afin de lui assurer une plus grande légitimité scientifique et de pointer la distance entre le paradigme keynésien, jugé empreint d'idéologie, et cette légitimité (et c'est à cette vision que la démarche de Sargent offre un contraste instructif) ${ }^{8}$.

\subsection{UNE NOUVELLE ARTICULATION ENTRE THEORIE ET MODELE}

Dans « Methods and Problems in Business Cycle Theory » (Lucas, 1980a), Lucas affirme :

A "theory" is not a collection of assertions about the behavior of the actual economy but rather an explicit set of instructions for building a parallel or analogue system a mechanical, imitation economy. (Lucas, 1981, 272)

La théorie fixe un cadre pour construire des modèles macroéconomiques. Plus précisément, ces modèles doivent respecter la « discipline » de l'équilibre général intertemporel et être microfondés afin de formuler les équations comportementales du modèle à partir de problèmes d'optimisation au niveau microéconomique. C'est le premier impératif de la construction de modèles pour Lucas : ces modèles doivent être en cohérence avec les principes de la théorie de l'équilibre général (TEG), telle qu'elle est formulée par Arrow et Debreu (voir Sergi, 2017, chapitre 1) ${ }^{9}$.

Lucas rompt avec la vision intuitive de l'articulation entre théorie et modèle, selon laquelle « une théorie est un ensemble de propositions sur la réalité » quand un modèle, lui, est conçu comme " une tentative d'expliciter rigoureusement les implications d'une certaine partie de la théorie » (De Vroey, 2015, 177). Dans cette optique, la théorisation n'est plus cette activité qui vise à mettre en ordre le monde, en proposant une grille de lecture permettant d'appréhender les lois et les mécanismes qui l'animent.

Pour Lucas, " une théorie s'occupe de constructions imaginaires », ce qui la conduit à être fondamentalement «non-réaliste » (ibid). Il en vient à penser que « la focalisation

\footnotetext{
${ }^{7}$ Il faudra par exemple attendre les travaux ultérieurs de Kydland et Prescott (1982) pour simuler des modèles dynamiques d'équilibre général visant à reproduire les fluctuations économiques.

8 Sur le lien entre idéologie et économie keynésienne, pour Lucas, voir De Vroey (2011).

${ }^{9}$ Chez Lucas, la TEG constitue véritablement un « camp de base ». Cette référence lui permet de s'inscrire dans la tradition de l'abstraction théorique considérée comme la plus rigoureuse, à une époque o\’u la TEG n'est pas encore réellement tombée en désuétude, suite au théorème de Sonnenschein-MantelDebreu. Sur cette base, Lucas peut alors critiquer les formalisations des équations des modèles keynésiens, jugées « ad hoc » et non fondées théoriquement.
} 
sur le "réalisme" d'un modèle économique subvertit son utilité potentielle pour penser la réalité » (Lucas, 1981, 271). Le réalisme d'un modèle, dans sa capacité à représenter le monde ou une partie du monde, n'est pas un critère adéquat d'évaluation de celui-ci. Ce qui doit intéresser le macroéconomiste, c'est la capacité de son modèle à nous fournir de " meilleures imitations » de la réalité (ibid. 272), entendues comme la reproduction quantitative des données observées ${ }^{10}$. Le modélisateur cherche à " imiter de près le comportement des séries temporelles de l'économie réelle » (ibid. 219). Statistiquement parlant, les simulations du modèle doivent faire apparaître des séries dont les premiers et seconds moments statistiques sont proches des moments des séries temporelles réelles, tout en retranscrivant avec précision les co-mouvements de ces différentes séries entre elles.

Cette volonté d'imitation fait émerger un mode de justification instrumentaliste des choix de modélisation : la capacité du modèle à reproduire les cycles du monde réel va guider la construction du modèle. Ainsi, Lucas justifie-t-il son économie d'archipel (Lucas, 1972) avec des marchés distincts situés sur différentes îles par la capacité de son économie modélisée à générer des variations cycliques :

The introduction of separate, informationally distinct markets is not a step toward "realism" or (obviously) "elegance" but, rather, an analytical departure which appears essential (in some form) to an explanation of the way in which business cycles can arise and persist in a competitive economy. (Lucas, 1981, 199)

C'est parce que ces îles distinctes permettent de justifier des restrictions d'information (les agents connaissent les prix sur leur île au temps $t$, mais pas sur les autres îles à la même période) et ainsi d'introduire des effets réels pour les chocs monétaires (générant alors de potentiels cycles économiques) qu'il s'agit d'une bonne « explication » des cycles économiques dans une "économie concurrentielle » (ibid.). Mais l'on voit bien que cette économie d'archipel n'a aucune prétention à passer le test d'une quelconque correspondance avec le monde réel, elle n'est qu'une simple fiction ${ }^{11}$.

Lucas ne simule pas son modèle de 1972, mais il constitue pour lui une première étape dans un projet plus global. A terme, il doit être possible de construire une économie analogique qui produise par simulation des cycles dont l'ampleur et la persistance ressemble aux fluctuations que l'on repère dans les économies réelles. Ce qui compte à terme pour Lucas, c'est l'adéquation des simulations du modèle avec les données observées. Peu importe que le modèle en lui-même soit réaliste, qu'il offre une représentation fidèle, ou $\mathrm{du}$ moins partiellement fidèle, ou, pourrait-on dire, vraisemblable, de la manière dont fonctionne une économie de marché, seul compte le résultat final. La construction du modèle est soumise à cet impératif (qui s'ajoute donc

\footnotetext{
${ }^{10}$ Dans une lettre à Ray Fair, daté du 2 octobre 1981, Lucas explique que quand il parle de « meilleures imitations », " "meilleures" signifie quelque chose comme "collant [fitting] au plus de faits possibles", ou "collant mieux aux faits", ou "passant plus de tests", ou quelque chose de la sorte » (Lucas, Archives, Box 4, Folder 1981 3/3, dans Sergi, 2017, Chapitre 1).

11 Sur le modèle de 1972, et ses développements ultérieurs, voir Hoover (1988, chapitres 2 et 3 ).
} 
au premier impératif de cohérence théorique). En cela on peut dès lors affirmer que Lucas revendique une forme d'instrumentalisme méthodologique : les hypothèses n'ont pas pour fonction d'être en adéquation plus ou moins complète avec la réalité et le modèle ne vise pas à offrir une représentation fidèle du processus sous-jacent au phénomène observé ; ne compte que l'adéquation (statistique) des prévisions du modèle avec la manifestation de ce même phénomène. Et c'est ce qui l'amène à considérer que toute critique sur le réalisme potentiel des hypothèses du modèle est vaine et inutile. Les hypothèses d'un modèle pour Lucas sont « a-réalistes » (Sergi, 2017, Chapitre 1) et, de ce fait, la question du réalisme de ces hypothèses est sans aucun fondement au vu de l'objectif assigné à la macroéconomie :

One can ask, for example, whether expectations are rational in the Klein-Goldberger model of the United States economy; one cannot ask whether people in the United States have rational expectations. (Lucas, Archives, Various. Box 23, Barro Folder, dans De Vroey, 2015, 177)

La vision idéalisée d'un modèle chez Lucas semble différer de la vision de Friedman. Mäki (2009b) défend de manière convaincante que Friedman s'inscrit dans la tradition du réalisme scientifique : les modèles économiques se réfèrent au monde réel, et ce dernier guide la construction des premiers. Mais, dans le même temps, les hypothèses d'un modèle sont nécessairement « abstraites » et « imprécises » (Friedman, 1953), elles constituent une « approximation » plus ou moins bonne de la réalité. En tant qu'approximation, elles sont nécessairement «fausses » et « irréalistes » (ibid.), et c'est pour cette raison qu'un modèle doit être jugé sur ses conclusions et non sur ses hypothèses. Chez Lucas, les hypothèses ne se réfèrent pas au monde réel, et elles ne peuvent donc être jugées selon un critère de correspondance à ce monde. Chez Lucas, les hypothèses, et tout particulièrement les anticipations rationnelles, sont " a-réalistes ». Dès lors, Lucas incarne une image plus fidèle que Friedman de l'instrumentalisme méthodologique, tel qu'il est défini par Mäki (1998).

Pour Lucas, l'économie modélisée et l'économie réelle doivent être clairement distinguées, et le seul pont qui peut exister entre les deux va des simulations de la première aux séries temporelles de la seconde ${ }^{12}$. Cette volonté de cloisonnement strict se justifie par le rôle unique que Lucas attribue à la macroéconomie : être capable de répondre à des questions de politique économique, et ce de manière pleinement scientifique (c'est-à-dire par l'utilisation d'un modèle robuste empiriquement). Quant à la capacité de la discipline à développer l'intelligence des phénomènes macroéconomiques et à en proposer un récit systématique, elle n'est plus une priorité.

\footnotetext{
12 Une métaphore peut ici être éclairante : imaginons la construction d'un robot « humain ». L'objectif des constructeurs est que le robot reproduise le plus fidèlement possible un certain nombre de mouvements $\mathrm{du}$ corps humain. Le robot sera une réussite plus ses actions sembleront se confondre avec celles d'un individu lambda. Cependant, le fait de démonter le robot pour étudier son fonctionnement ne vous dira pas grand-chose sur la manière dont fonctionne le corps humain. Autrement dit, le fonctionnement sousjacent du robot doit être séparé de celui du corps humain.
} 


\subsection{LE MODELE MACROECONOMIQUE COMME INSTRUMENT DE POLITIQUE ECONOMIQUE}

Pour Lucas, " le progrès dans la pensée économique consiste à obtenir de meilleurs modèles d'économies abstraites analogues, et non à produire de meilleures observations verbales sur le monde " (Lucas, 1981, 276, je souligne). Il définit explicitement la tâche du macroéconomiste tel qu'il l'imagine, dans un futur proche :

Our task as I see it is to write a FORTRAN program that will accept specific economic policy rules as "inputs" and will generate as "output" statistics describing the operating characteristics of times series we care about, which are predicted to result from these policies. (Ibid. 288)

Le macroéconomiste construit son modèle en respectant un certain nombre de "principes architecturaux », les fameuses « instructions » de Lucas, assurant la validité théorique du modèle. Tout particulièrement, il doit veiller à microfonder les équations comportementales de son modèle, afin de prémunir ce dernier contre la fameuse critique de Lucas (Lucas, 1976). ${ }^{13}$. Une fois le modèle bâti, on vérifie que les simulations du modèle produisent des résultats en accord avec les données de l'économie réelle. Cette concordance assurée, on peut juger le modèle robuste et l'utiliser pour l'évaluation de politique économique. On entre dans ce modèle différentes règles de politiques économiques, et l'on regarde, après simulation, laquelle produit les résultats économiques les plus appréciables ${ }^{14}$.

Le macroéconomiste se transforme alors, en quelque sorte, en technicien chargé d'élaborer un outil technique, un « logiciel de politique économique », mis au service des institutions publiques chargées de la politique économique. Lucas s'éloigne ainsi de la figure de l'économiste public, conseiller des princes, dont Keynes et Friedman sont les exemples. La tâche du macroéconomiste n'est plus de développer des outils de compréhension du monde, de proposer une interprétation de la conjoncture économique, et d'offrir des solutions pragmatiques à certains problèmes économiques. Conséquence de cette conception instrumentaliste du travail scientifique, la théorie économique n'a plus vocation à mettre en ordre le monde et à expliquer les phénomènes qui s'y manifestent. En poussant la logique épistémologique de Lucas jusqu'à son paroxysme, il devient clair que le rôle explicatif du travail de l'économiste passe au second plan.

Donner une explication d'un phénomène économique particulier, «c'est citer ses causes ; (...) c'est décrire le mécanisme causal qui en est responsable » (Reiss, 2013, 120). Bien entendu, il est possible de distinguer des chaînes de causalité au sein des modèles de la $\mathrm{NEC}$, d'observer comment telle hypothèse conduit à telle conséquence économique au

\footnotetext{
${ }^{13}$ L'évaluation des politiques économiques n'est possible que si l'on prend en compte le fait que les agents réagissent aux changements de ces politiques, et il est donc nécessaire pour cela de prendre en compte la manière dont ils anticipent ces politiques et dont ils changent leur comportement en fonction de ces anticipations.

${ }^{14}$ Pour Lucas, les politiques discrétionnaires, à l'inverse des politiques de règles, font entrer l'analyse économique dans le domaine de l'incertitude, au sens de Knight, et empêche toute " évaluation quantitative scientifique» des politiques économiques (Lucas, 1981,125) ; voir aussi infra, section 1.3).
} 
sein du modèle, mais du fait de la frontière que Lucas voudrait instaurer entre le modèle et le monde réel, ces chaînes de causalité n'ont pas vocation à revêtir une pertinence quelconque au-delà du modèle lui-même. Les enchaînements causaux du modèle n'ont aucune légitimité explicative en dehors du cadre du modèle ; ils ne se réfèrent pas au monde réel. Seul compte le résultat final du mécanisme causal, que l'on compare avec les observations du monde réel.

Dès lors, pour Lucas, l'objectif du macroéconomiste n'est pas d'expliquer des événements historiques particuliers ${ }^{15}$. La stagflation que connaissent les Etats-Unis dans les années 1970 n'est donc pas un phénomène singulier auquel il faut fournir une explication ${ }^{16}$. Lucas met d'ailleurs en garde les macroéconomistes contre la tentation de répondre aux sirènes de la demande sociale et de construire des théories ad hoc à cet effet :

To the journalist, each year brings unprecedented new phenomena, calling for unprecedented new theories (where "theory" amounts to a description of the new phenomena together with the assertion that they are new). Since there is an obvious sense in which this view carries some truth, (...) I have argued elsewhere that it is in our interest to take exactly the opposite viewpoint in the study of business cycles, or as close to an opposite view as we can get by with and will maintain this attitude below. (Lucas, 1981, 273)

Se dégage ainsi la vision d'un économiste en surplomb, détaché des événements courants et de la conjoncture présente, et avec pour tâche d'aboutir, dans le futur, à la construction d'une théorie générale des cycles d'affaires dont les modèles joueront le rôle de laboratoire à des fins de simulation de différentes politiques économiques. Pour Lucas, la macroéconomie doit reconquérir son autonomie et développer des bases théoriques solides sur lesquelles il sera possible de bâtir un modèle guidant scientifiquement (et mécaniquement) l'intervention politique ${ }^{17}$.

\subsection{L'INSTRUMENTALISME METHODOLOGIQUE EN TANT QUE STRATEGIE RHETORIQUE}

Cette posture méthodologique de Lucas ne relève pas uniquement de profondes convictions épistémologiques, mais elle est aussi le fruit d'un contexte académique

\footnotetext{
${ }^{15}$ Kyun $(1988,111)$ montre bien le tournant sémantique qui s'opère autour du concept de « cycle » entre les travaux fondateurs de Mitchell et du NBER, et ceux de la NEC. L'objectif n'est plus d'isoler des cycles économiques, d'en distinguer les phases de "prospérité », de "crise », et de "récession » (Mitchell, 1923), mais plutôt d'offrir une théorie générale des fluctuations économiques, dans laquelle les cycles économiques sont vus comme « tous identiques » (Lucas, 1981, 218).

16 Il est important de noter néanmoins que, dans le travail de Lucas, la Grande Dépression des années 1930 semble occuper une place particulière. Lucas y consacre d'ailleurs un article où, avec Rapping, il tente d'appliquer leur modèle, développé un peu plus tôt, pour expliquer le niveau du chômage à l'époque (Lucas et Rapping, 1969 ; 1972). La Grande Dépression constitue un phénomène à part pour Lucas, que la théorie générale des cycles qu'il veut construire n'a pas pour vocation d'expliquer (De Vroey, 2015, 152 ; 165).

${ }^{17}$ Ainsi, l'objectif des macroéconomistes est d'élaborer des règles pour les politiques monétaires et budgétaires que les autorités publiques pourront appliquer.
} 
particulier : la déstabilisation des macroéconomistes face à la stagflation et les batailles pour imposer un point de vue au sein, ainsi qu'à l'extérieur, du champ de la discipline. Comme l'explique Mäki, l'instrumentalisme comporte une dimension stratégique :

One of the benefits of instrumentalism is that it facilitates dealing with theories or assumptions which are somehow puzzling, such as being counterintuitive, or as being contradicted by experiments or by other theories. The instrumentalist solution is to recommend a relaxed attitude towards such theories: it does not matter that theories appear to include such puzzling features; once we understand that theories are not intended as factual claims about reality, those features no longer appear as puzzling. (Mäki, 1998, 254)

En revendiquant une forme d'instrumentalisme, Lucas cherche à éloigner le regard de l'irréalisme potentiel des hypothèses formulées dans ses modèles. Or c'est l'angle d'attaque principal choisi par les économistes keynésiens pour discréditer les travaux de la NEC. Dans son entretien avec Klamer, Modigliani met en doute la pertinence de l'hypothèse selon laquelle les travailleurs forment leurs anticipations de manière rationnelle (Klamer, 1984, 125-26). Ce procès en réalisme se retrouve sous la plume de Solow $(1978)$ ou de Tobin $(1980 ; 1981)$. En séparant clairement l'économie modélisée de l'économie réelle, et en clamant que parler d'anticipations rationnelles pour des individus réels n'a pas de sens, Lucas exclut d'office les arguments des keynésiens contre l'hypothèse d'anticipations rationnelles. Le modèle doit être jugé sur les résultats qu'il produit et rien ne sert d'engager le débat sur le réalisme des anticipations rationnelles.

Cependant, cette volonté de cloisonner les anticipations rationnelles dans la sphère du modèle n'est pas sans failles, et quelques ambiguïtés apparaissent dans les propos de Lucas. Ainsi s'évertue-t-il parfois à justifier leur utilisation dans une perspective qui tend vers une forme de réalisme scientifique, c'est-à-dire dans une perspective qui justifie qu'il peut être approximativement vrai de supposer que les individus dans l'économie réelle forment leurs anticipations de manière rationnelle :

These considerations [sur la distinction de Knight entre risque et incertitude, A.G.] explain why business cycle theorists emphasized the recurrent character of the cycle, and why we must hope they were right in doing so. Insofar as business cycles can be viewed as repeated instances of essentially similar events, it will be reasonable to treat agents as reacting to cyclical changes as "risk", or to assume their expectations are rational, that they have fairly stable arrangements for collecting and processing information, and that they utilize this information in forecasting the future in a stable way, free of systematic and easily correctable biases. (Lucas, 1981, 224)18

Puisque les cycles se répètent, par définition, on peut supposer que les agents en connaissent les mécanismes, et construisent donc plus ou moins consciemment un modèle de cycles qu'ils utilisent pour anticiper. Lucas entend ainsi trouver une

\footnotetext{
${ }^{18} \mathrm{~L}$ 'expression « to treat agents as reacting » nous renvoie ici au as-if friedmanien. Au vu des caractéristiques des cycles économiques, il est « raisonnable » de considérer que les agents forment leurs anticipations de manière rationnelle (au sens de Muth, 1961), même si cette hypothèse n'est pas vraie dans le sens où elle constitue une idéalisation, une approximation.
} 
légitimation réaliste à l'hypothèse d'anticipations rationnelles et semble alors lui-même relâché sa position méthodologique rigide, comme le montre Sergi (2017, Chapitre 1), à travers l'étude de l'usage qu'il fait du terme d'analogie. L'élaboration d'un modèle implique d'engager des discussions en termes de réalisme et de vraisemblance, sur les matériaux choisis pour sa construction, d'autant plus quand celui-ci a pour but, en dernière instance, de servir d'instrument à la politique économique. On pourrait en fait affirmer que Lucas défend un instrumentalisme " modéré ${ }^{19}$. La question du réalisme des hypothèses est une question de second ordre, elle est même un fardeau face à ses opposants. Mais cela ne signifie pas pour autant qu'elle ne revêt pas un minimum de sens dans son esprit. Il revendique simplement la possibilité de le laisser développer ses modèles librement (c'est-à-dire sans remettre en question constamment les hypothèses fondamentales du modèle), la fin devant, à long terme, justifier les moyens employés.

Cette stratégie doit permettre de se débarrasser de la question du réalisme des hypothèses et de porter l'attention sur les faiblesses des modèles keynésiens, attaqués par Lucas et Sargent pour leur absence de fondement théorique et leur caractérisation «a priori » des variables pertinentes, de leur exogenéité ou endogenéité, et des termes d'erreurs (Lucas et Sargent, 1979, 4-5)20.

Si la position de Sargent est intéressante, c'est justement parce que ce dernier, à travers des textes publiés au début des années 1980, opte pour une stratégie méthodologique différente face aux keynésiens, qui vient en supplément de l'instrumentalisme modéré de Lucas. Sa démarche s'appuie sur une forme de réalisme scientifique qui est absent des propos de Lucas, et qui le conduit à donner un sens différent aux hypothèses d'optimisation des agents et d'anticipations rationnelles.

\section{UNE AUTRE AMBITION POUR LES ANTICIPATIONS RATIONNELLES]\{UNE AUTRE AMBITION POUR LA THEORIE DES ANTICIPATIONS RATIONNELLES}

Dans Rational Expectations and Inflation, Sargent affiche une ambition qui est absente des travaux de Lucas : celle de développer une "théorie des anticipations rationnelles » qui soit un outil heuristique pertinent pour offrir une grille de lecture à un certain nombre d'événements historiques et bâtir un nouveau langage pour traiter des phénomènes économiques. Le choix des cas historiques qu'étudie Sargent n'est pas anodin: ils concernent tous des expériences particulières d'inflation. Ce choix est sans doute d'abord lié au fait que c'est ce type d'expériences que les anticipations

\footnotetext{
${ }^{19}$ Selon Mäki, « a more moderate version of instrumentalism says that theories do possess such semantic properties, but that these properties are irrelevant, the relevant properties being pragmatic. According to this version, it does not matter even if the terms of a theory fail to refer to anything real (even though they might so refer) or if the claims of a theory are false (even though they might be true) » (Mäki, 1998, 254, je souligne).

${ }^{20}$ Goutsmedt (2017) étudie le contexte de la présentation de cette critique par Lucas et Sargent, ainsi que sa réception par ceux à qui elle s'adresse, lors de la conférence de 1978, organisée par la Federal Reserve Bank de Boston.
} 
rationnelles sont les plus aptes à décrire. Ces expériences historiques constituent donc des faire-valoir. Mais ce choix est aussi lié au contexte de publication: la question du niveau d'inflation et des politiques de désinflation est centrale dans les débats aux EtatsUnis au début des années 1980. Pour Sargent, il est urgent d'appliquer la logique des modèles de la NEC aux problèmes contemporains des économies occidentales, afin de prouver qu'il est possible de faire concurrence aux keynésiens sur ce terrain-là.

Comme mentionné en introduction, je m'intéresse aux quatre premiers articles de l'ouvrage. Sargent a poursuivi cette tâche dans des travaux ultérieurs (publiés dans les éditions suivantes du recueil) en s'intéressant, par exemple, à la Révolution française ou à la situation économique de Hong Kong dans les années 1980, et en mobilisant des outils conceptuels et techniques développés par la suite. Je me limite à la première moitié des années 1980 pour plusieurs raisons : ces travaux de Sargent répondent à une logique de promotion de la NEC au sein du champ de la macroéconomie (alors que les idées de la NEC sont solidement installées dans la discipline par la suite) ; ces travaux ont pour ligne de mire l'explication de la stagflation, les politiques nécessaires pour en sortir, et le coût de ces politiques ${ }^{21}$. Dans son discours de lauréat du prix de la Banque de Suède en sciences économiques en mémoire d'Alfred Nobel, il propose une synthèse explicite de ses influences :

I work in a macroeconomic tradition developed by John Muth, Robert E. Lucas Jr., Edward C. Prescott, Finn Kydland, Nancy Stokey, and Neil Wallace. I use macroeconometric methods championed by Lars Peter Hansen and Christopher A. Sims. I interpret macroeconomic history in ways advanced by Irving Fisher, Milton Friedman, Anna Schwartz, and François Velde. (Sargent, 2012, 1-2)

Cette « théorie des anticipations rationnelles » (Sargent, 2013, xxii) qu'il cherche à bâtir s'inscrit dans l'esprit de la « tradition macroéconomique » de la NEC. Elle repose sur le principe selon lequel les individus sont des individus optimisateurs et s'efforcent d'anticiper les politiques économiques futures. Ce principe, jugé intuitif et de bon sens par Sargent, revêt un caractère réaliste, aux antipodes de l'a-réalisme prôné par Lucas, et réside au fondement d'une théorie de l'inflation qui permet d'analyser un grand nombre de cas historiques particuliers.

\subsection{UN LANGAGE A PORTEE PRATIQUE}

Comparant l'approche des nouveaux classiques à celle de l'analyse keynésienne, De Vroey considère qu'alors que la seconde est « simple à comprendre », peu technique, le

\footnotetext{
${ }^{21}$ Il est intéressant de noter que ces travaux, ainsi que le recueil de manière générale, sont assez peu connus des historiens de la macroéconomie. Ainsi, alors même qu'elle consacre un livre à la trajectoire de Sargent, Sent (1998) semble laisser de côté cette partie de sa carrière. En se concentrant sur la relation entre « théories économiques » et «méthodes économétriques » chez Sargent, elle occulte le travail de ce dernier pour connecter la théorie avec des événements économiques réels, et ainsi justifier l'usage des anticipations rationnelles.
} 
coûut d'entrée pour la première est plus élevé, car faisant appel à " de nouvelles techniques mathématiques complexes » (De Vroey, 2015, 190). Par conséquent, la " conception Lucasienne de la théorie et des modèles est contre-intuitive et de peu d'attrait pour le profane », tout à l'inverse de la macroéconomie keynésienne qui a " fait son chemin dans les journaux et les discours politiques » (ibid.).

Sargent semble partager le même point de vue dans l'avant-propos de la première édition de Rational Expectations and Inflation, écrit en 1985. Sargent y souligne justement la portée pratique de l'analyse keynésienne vis-à-vis de sa rivale. La force du paradigme keynésien est d'avoir développé, à travers le modèle IS-LM, entre autres, un langage qui lui permet d'interpréter de manière intuitive la conjoncture économique et facilite la communication entre macroéconomistes, ainsi qu'avec les décideurs dans les institutions de politique économique :

During the long period of dominance of Keynesian macroeconomic theory, there developed a broad body of practical experience in applying the theory quickly in informal contexts. Such experience created ease of application and communication, which were important ingredients in the practical success of Keynesian economics as an applied theory. (Sargent, 2013, xxi)

Les modèles avec anticipations rationnelles sont, sans conteste, d'une appréhension plus difficile pour les non-initiés, du fait de leur « orientation hautement technique » (ibid.). Les anticipations rationnelles impliquent de nouveaux concepts, un « nouveau langage » (ibid.), et remettent ainsi totalement en question la pratique quotidienne de la macroéconomie. Sargent estime que c'est là l'une des raisons des difficultés de la NEC, dans les années 1970 et au début des années 1980, à s'imposer dans le champ de la macroéconomie:

One of the (legitimate) reasons for resistance to rational expectations ideas in macroeconomics has been that they are perceived as destroying the old language and tools of applied macroeconomics while providing no workable substitute. (Ibid.) ${ }^{22}$

En montrant les limites du consensus keynésien et en contribuant à son renversement, la NEC discréditerait en fait la capacité des macroéconomistes à produire un discours sur le réel et à conseiller les autorités publiques. Dès lors, Sargent comprend bien que si la NEC veut mener à bien la révolution méthodologique et théorique qu'elle a entreprise, elle se doit de se substituer à l'analyse keynésienne et de remplir les mêmes tâches. C'est tout l'enjeu des articles republiés dans Rational Expectations and Inflation, auxquels Sargent donne une cohérence globale à travers cet avant-propos :

\footnotetext{
${ }^{22}$ Quelques années plus tard, cette question demeure et Krugman semble partager le même diagnostic, vantant l'utilité du modèle IS-LM face aux modèles dits " microfondés ». Même si les modèles de type ISLM sont moins complets et plus « $a d h o c »$, ils demeurent pour Krugman un outil important de pensée dans les institutions publiques et permettent de raisonner plus facilement sur des questions de politique économique, tout en offrant des réponses qui ne sont que rarement absurdes et erronées (Krugman, 2000, 41).
} 
My main reason for writing this book was to try my hand at putting rational expectations macroeconomics to work at an informal, non-econometric level in order to describe and interpret some recent and historical economic events. (Sargent, 2013, xxi)

Il reprend ce point plus loin, dans le premier article du recueil :

The practical success of the rational expectations approach to macroeconomics perhaps ultimately depends on whether it can become a routine device that can be used to think about macroeconomic problems informally and on the backs of envelopes. (Ibid. 14)

Dans un premier temps, Sargent s'affirme clairement comme partie prenante du projet de Lucas. Il s'agit de reprendre le projet initial des keynésiens, en construisant des modèles économétriques pour « offrir des conseils quantitatifs sur les effets de différentes politiques économiques » (ibid. 1). Mais ces modèles doivent prendre en compte que, dans leurs décisions, les agents privés intègrent les règles de décision des gouvernants, et, dès lors, si ces règles changent, les agents privés adapteront leur comportement. L'objectif est donc de construire des modèles capables d'estimer quelle sera la réaction des agents économiques quand les règles fixées par les institutions de la politique économique et leurs décisions changent. Cependant, Sargent reconnaît qu'il s'agit d'une tâche extrêmement « ambitieuse » et que le chemin est encore long pour obtenir « un modèle approprié pour analyser un certain nombre de questions intéressantes sur la politique macroéconomique » (ibid. 13). C'est pour cette raison qu'en parallèle, il propose de recourir à une méthode alternative s'ajoutant au projet de long terme qu'est la construction d'un tel modèle :

But we are still some way from a model suitable for analyzing many interesting questions of macroeconomic policy. This is one reason that it is useful to pursue alternative styles of analysis that try to reflect the basic principle that private agents' behavior depends on the rules of the game set by the government. This brings us to economic history. (Ibid. 13)

Sargent reprend alors le vocabulaire de Lucas (que ce dernier appliquait pour les modèles formels qu'il désirait développer) et considère que les événements historiques auxquels il s'intéresse constituent des « laboratoires pour l'étude des changements de régime [de politique économique] » (ibid. 40). Avec ces cas historiques, la quantité de données est insuffisante pour avoir des séries temporelles de qualité, à utiliser dans les modèles économétriques. De manière générale, ces derniers n'étant pas encore abouti, Sargent leur substitue (pour les travaux mis en avant ici) une pratique plus intuitive, mais donc moins formalisée et non testée économétriquement.

Sargent crée ainsi une brèche dans la séparation stricte que fait Lucas entre l'économie modélisée et l'économie réelle. D'un côté, il semble reconnaître la nécessité de cette 
séparation, comme le suggère son entretien avec Klamer (1984) ${ }^{23}$. Mais de l'autre, avec ce « style alternatif d'analyse », il s'émancipe de cette distinction, tout en ayant conscience de la perte de rigueur scientifique qui pourrait en échoir :

To the extent that these less formal methods require more judgment, discretion, and cleverness from the analyst, they are in a sense less reproducible and automatic than are the formal methods. (Sargent, 2013, 14)

A travers cette démarche, l'hypothèse d'anticipations rationnelles endosse un nouveau statut. Elle n'est plus seulement une hypothèse a-réaliste assurant la validité interne du modèle, mais sa signification se retrouve projetée dans le monde réel. Les anticipations rationnelles constituent dès lors une manière de décrire le comportement des agents économiques réels (elles ne sont plus circonscrites au comportement des agents du modèle). Et de ce comportement découle tout un ensemble de conséquences macroéconomiques que la «théorie des anticipations rationnelles » permet, en principe, d'expliquer.

\subsection{LES PRINCIPES FONDAMENTAUX DE LA « THEORIE DES ANTICIPATIONS} RATIONNELLES »

Selon Sargent, deux principes sont au coeur de Rational Expectations and inflation : «l'interdépendance stratégique » et la nécessité de la coordination entre politique monétaire et politique budgétaire (Sargent, 2013, xxii). De manière générale, les agents économiques forment leurs décisions (de consommation, d'investissement, etc...) en fonction de leur environnement (de manière à optimiser des fonctions-objectifs). Si un élément de cet environnement évolue, ils optimisent de nouveau en fonction de ce nouvel environnement. Dès lors, et c'est le sens même de la critique de Lucas, tout changement dans la manière dont les différentes politiques économiques sont menées conduit les agents à modifier leur comportement. Les institutions qui régissent les politiques économiques doivent donc prendre en compte la réaction des agents face à un changement de politique économique (d'où l'interdépendance entre autorités publiques et agents privés). C'est cette idée intuitive qui justifie l'utilisation de l'hypothèse d'anticipations rationnelles. Sargent choisit ainsi un mode de justification des anticipations rationnelles qui se veut ancré dans une forme de réalisme scientifique, et ce afin de légitimer son utilisation en montrant son apport potentiel dans l'interprétation d'un ensemble de phénomènes.

De cette intuition initiale découle une certaine théorie de l'inflation. Le modèle formel que Sargent a en tête dérive du modèle de l'article de \citet\{cagan1956\} sur

\footnotetext{
${ }^{23}$ Quand on lui demande s'il considère que les politiques de stabilisation actives sont inefficaces, tout comme dans le modèle théorique élaboré avec Neil Wallace (1976), Sargent n'hésite pas à limiter ce résultat au domaine du modèle : " I'd like to distinguish sharply between statements that Neil and I made about particular models, and statements that we make about policy and the world right now. I certainly wouldn't endorse the proposition that any policy doesn't matter and is ineffective » (Klamer, 1984, 70).
} 
l'hyperinflation allemande, auquel il ajoute l'hypothèse d'anticipations rationnelles. La demande d'encaisses réelles dépend de leur valeur future anticipée et prend la forme suivante :

$$
\frac{M_{t}}{p_{t}}=\alpha-\beta E_{t}\left[\frac{p_{t+1}}{p_{t}}\right] \text { avec } \alpha>\beta \geq 0
$$

avec $M_{t}$ le stock de monnaie Banque Centrale au temps $t$, $p_{t}$ le niveau des prix, et $E_{t}[\cdot]$ la valeur de [.] anticipée par les agents économiques au temps $t$. Dans cette équation, la demande de monnaie est une fonction décroissante de l'inflation anticipée, $E_{t}\left[\frac{p_{t+1}}{p_{t}}\right]$. Après quelques transformations (voir Sargent, 2013, 24), on obtient l'expression suivante pour l'inflation :

$$
p_{t}=\frac{1}{\alpha} \sum_{j=0}^{\infty}\left(\frac{\beta}{\alpha}\right)^{j} E_{t} M_{t+j}
$$

Le niveau des prix est une fonction de l'offre de base monétaire anticipée aujourd'hui et pour toutes les périodes à venir. Ainsi, l'inflation est déterminée par la perception que les agents ont de la politique monétaire future. Si les agents pensent que la Banque Centrale va augmenter la création monétaire, alors les prix augmenteront aujourd'hui (quelle que soit l'action effective de la Banque Centrale). Dès lors, l'inflation peut être relativement élevée simplement parce que les agents s'attendent à une politique monétaire expansionniste.

De manière générale, la perception qu'ont les agents à l'égard de l'action des autorités publiques dépend selon Sargent du « régime de politique économique » choisi. Ce qui renvoie au deuxième principe fondamental avancé par Sargent, celui de la nécessaire coordination entre politique monétaire et politique budgétaire. A un instant $t$, le déficit budgétaire n'est pas nécessairement relié à la création de monnaie Banque Centrale. En effet, un déficit peut être financé par l'émission de titres de dette d'Etat (ibid. 25). Cependant, plus ce déficit se répète à chaque période, plus il est probable qu'il finisse par être financé par de la création monétaire, ce qui, d'un point de vue monétariste, générerait de l'inflation.

Dès lors, la question des anticipations et de la perception des agents face au « régime de politique économique » intervient de nouveau. Dans le cadre de « l'arithmétique de la contrainte budgétaire du gouvernement » (ibid. 143), tout déficit budgétaire récurrent, qui accroît donc la dette de l'Etat, devra être compensé dans le futur soit par une hausse des recettes et une baisse des dépenses publiques, soit par de la création monétaire. Si les agents anticipent que le premier cas prévaudra, alors nous nous trouvons dans le cadre de «l'équivalence ricardienne ». Si les agents pensent que les déficits budgétaires conduiront à une politique monétaire expansionniste dans le futur, alors les déficits budgétaires créeront une tendance inflationniste dès aujourd'hui. Face aux déficits présents, les agents anticipent une création de monnaie supplémentaire dans le futur, ce qui stimule l'inflation aujourd'hui. Une politique monétaire restrictive qui vise à ralentir 
l'inflation ne pourra fonctionner que si elle est coordonnée avec la politique budgétaire, c'est-à-dire s'il n'y a pas de déficits budgétaires. En effet, les déficits impliqueraient que la politique monétaire ne pourrait pas être maintenue dans le temps, ce qui annulerait ses effets et ruinerait les efforts de désinflation, tout en plongeant l'économie concernée dans la récession.

A partir de l'idée que les agents économiques prennent en compte les changements de politiques économiques et anticipent leurs variations dans le futur, Sargent dégage un ensemble de conséquences qui permettent d'interpréter les variations de l'inflation (et dans une certaine mesure celles du chômage et de la croissance). Ses deux principes fondamentaux lui offrent une grille de lecture pour appliquer cette théorie des anticipations rationnelles à des expériences économiques particulières. Mais cette grille de lecture est aussi l'occasion de développer un discours sur les événements présents et sur les moyens de sortir de la « crise des années $1970 »$.

\section{LA THEORIE DES ANTICIPATIONS RATIONNELLES EN PRATIQUE}

Comme expliqué ci-dessus, le premier objectif de Sargent est de tester sa grille de lecture face à des événements historiques. Cette grille de lecture doit permettre de juger de l'efficacité ou non de certaines politiques économiques. Pour Sargent, c'est là l'occasion de se situer (et de situer la NEC) dans l'espace des prises de positions de politique économique au tout début des années 1980.

\subsection{INTERPRETER DES EVENEMENTS PARTICULIERS}

Dans "The Ends of Four Big Inflations » (l'article le plus cité et connu du recueil), Sargent s'intéresse aux mesures qui ont permis de mettre fin avec succès à une explosion de l'inflation, dans plusieurs pays d'Europe dans les années 1920. L'intérêt de ces mesures est qu'elles ont permis de stopper la croissance de l'inflation sans imposer dans le même temps à la société un coût élevé en termes de chômage et de chute de la production. Sargent s'intéresse ainsi à ce qu'on appelle dès les années 1970 le « ratio de sacrifice ", c'est-à-dire les conséquences en termes de chômage et de croissance d'une baisse d'un point du taux d'inflation ${ }^{24}$.

L'idée de Sargent est qu'une politique de désinflation sera efficace et peu coûteuse si elle parvient à être crédible auprès des agents économiques. Comme le montrent les équations de la section précédente, l'inflation dépend des anticipations des agents vis-àvis de la politique monétaire future. Si les agents croient que la politique monétaire sera

\footnotetext{
${ }^{24}$ Moins les prix et les salaires s'ajustent rapidement suite à une récession, plus la désinflation risque de prendre du temps, et donc la récession d'être coûteuse en termes d'emploi et de production
} 
en effet restrictive dans le futur, alors l'inflation se réduira dès aujourd'hui. Mais Sargent met en garde contre la tentation de croire, dans ce cadre d'analyse, qu'une politique de désinflation est aisée à mener à bien :

This is not to say that it would be easy to eradicate inflation. On the contrary, it would require far more than a few temporary restrictive fiscal and monetary actions. It would require a change in the policy regime: there must be an abrupt change in the continuing government policy, or strategy, for setting deficits now and in the future that is sufficiently binding as to be widely believed. (Sargent, 2013, 40)

Une politique de désinflation n'a aucune chance de réussir si elle repose sur des actions « isolées » (on retrouve ici l'idée de coordination entre politiques monétaire et budgétaire) et si ces actions sont "temporaires », ou considérées comme telles. La désinflation sera d'autant moins coûteuse que l'action du gouvernement apparaîtra « résolue et évidente » (ibid.). Le gouvernement doit donc fixer une stratégie cohérente et durable de lutte contre l'inflation, afin que cette stratégie soit jugée crédible par les agents et influe positivement sur leurs anticipations. Et si les politiques de désinflation mises en place par l'Autriche, la Hongrie, la Pologne et l'Allemagne ont été une réussite, c'est parce que ces politiques ont été jugées crédibles ${ }^{25}$.

Dans « Stopping Moderate Inflations: The Methods of Poincaré and Thatcher », Sargent entre dans le détail des prérequis à la réussite d'une politique de désinflation. Si ces prérequis sont satisfaits, alors cela implique que « le remède à l'inflation n'est pas coûteux » ou encore qu'on " peut attendre que les théorèmes de neutralité de la théorie approximent la réalité » (ibid. 116, je souligne). Autrement dit, si la situation économique étudiée respecte un certain nombre de critères, alors le théorème de neutralité de la monnaie, tel qu'exposé chez (Lucas, 1972), est valide, et les agents anticipent les effets de cette politique de désinflation et ajustent leur comportement par avance. Celle-ci n'aura donc pas d'effets sur les variables réelles. On voit bien à travers cette citation que Sargent s'écarte des précautions de Lucas, dans l'utilisation qu'il fait des modèles à anticipations rationnelles. A travers son étude de certaines situations économiques, il cherche à établir dans quels cas ces modèles sont valables. Dans ce type d'analyse, le décloisonnement entre économie modélisée et économie réelle est évident.

Que nous dit Sargent sur ces prérequis ? L'expérience du « miracle Poincaré » en France en juillet 1926 lui sert de point de référence. Alors que l'inflation demeurait à des niveaux élevés depuis 1923 et que le franc ne cessait de perdre de sa valeur, Raymond Poincaré, tout juste devenu président du conseil, a réussi à réduire l'augmentation des prix à un niveau soutenable. Pour cela, il a d'abord pu s'appuyer sur un large consensus politique : tous les partis politiques, exceptés les socialistes et les communistes, se sont rangés derrière lui (Sargent, 2013, 118). De plus, selon Sargent, la société française affichait un consensus relativement large sur les facteurs économiques de la

${ }^{25}$ Sargent souligne d'ailleurs que l'inflation a diminué alors même que la quantité de monnaie créée continuait de croître dans des proportions importantes (ibid. 42). 
dépréciation du franc et de l'inflation (" des déficits budgétaires persistants et la pression qui en résulte pour monétiser la dette publique », ibid.), mais aussi sur la manière de les stabiliser (« augmenter les impôts et réduire les dépenses publiques pour équilibrer le budget, tout en limitant fermement le montant de dette publique que la Banque de France peut monétiser », ibid.). Ces préconditions formaient ainsi un terreau propice à la réussite de la politique désinflationniste de Poincaré :

The French stabilization thus seems to fit the predictions of the rational expectations equilibrium approach. To the extent that it does fit, one reason is probably the high degree of political and intellectual consensus that prevailed at the time. (Ibid. 121)

Peut-on transposer cette analyse à la Grande Bretagne de Margaret Thatcher ? Pour Sargent, la situation est ici plus compliquée. Le large consensus que connaît la France en 1926 est en « grande partie le résultat de circonstances historiques » (ibid. 123), et il n'est pas sûr qu'il en aille de même pour la Grande Bretagne. Or l'analyse en termes d'anticipations rationnelles " suggère qu'un premier ministre ou même un parti politique uni n'a pas le pouvoir de créer les circonstances requises pour mettre fin à l'inflation rapidement et sans coûts » (ibid. 115) ${ }^{26}$. Autrement dit, l'administration Thatcher est dépendante des expériences économiques passées, et du fait qu'elle « arrive au pouvoir après vingt années de politiques de stop and go » (ibid.). A l'époque où Sargent écrit, Thatcher se heurte à l'opposition du parti travailliste et du parti socialdémocrate. Les deux camps ne partagent pas le même diagnostic et il n'existe pas « de large consensus sur la théorie » (ibid. 116). Les conditions économiques, sociales et politiques auxquelles fait face l'administration Thatcher poussent Sargent à exprimer de sérieux doutes sur sa capacité à réussir à réduire l'inflation :

For all of these reasons, it is difficult to interpret Thatcher's policy actions in terms of the kind of once-and-for-all, widely believed, uncontroversial, and irreversible regime change that rational expectations equilibrium theories assert can cure inflation at little or no cost in terms of real output. (Ibid.)

Les doutes qu'expriment Sargent sur la réussite potentielle d'une telle politique de désinflation ne s'arrêtent pas là. La politique de Thatcher, tout comme celle de Reagan, sont condamnées à l'échec, car elles ignorent l'un des principes fondamentaux de la théorie des anticipations rationnelles : la coordination entre politique monétaire et politique budgétaire.

Ce principe conduit en effet Sargent à considérer, dans " Reaganomics and Credibility », que la politique du président américain n'a que peu de chance de réussir à diminuer l'inflation sans coûts pour la croissance et le chômage. En effet, en 1981, l'administration Reagan met rapidement en place des réductions d'impôts, tout en annonçant des

\footnotetext{
${ }^{26}$ Sargent ajoute : «Whether or not the stage is set for successfully implementing a significant new policy regime is the result of intellectual and historical forces that individual political figures influence only marginally $\gg($ ibid.)
} 
réductions dans les dépenses publiques, qui ne viendront que plus tard. Le déficit budgétaire risque alors de se creuser selon Sargent, ce qui va à l'encontre de la politique monétaire restrictive menée par Paul Volcker à la FED. Les deux politiques entrent donc en conflit, et il y a une possibilité pour que les agents considèrent que la FED va être obligée d'assouplir sa politique en monétisant une partie des déficits de l'Etat fédéral, mettant alors en échec le succès de la politique de désinflation :

In this interpretation Reaganomics was not credible because it was not feasible. It was simply not feasible simultaneously to carry out both the fiscal and the monetary aspects of Reaganomics. Therefore, to rational observers, Reaganomics was incredible. (Ibid. 35)

A travers l'analyse de ces expériences économiques historiques ou présentes, Sargent entend justifier l'utilité de la théorie des anticipations rationnelles. L'idée d'agents économiques qui optimisent et qui forment leurs anticipations de manière rationnelle n'est plus alors réduite à n'exister qu'en tant que « simple instruction » permettant de construire un modèle. Elle a vocation à être vraisemblable, à dire quelque chose sur le monde. Elle résulte d'une intuition qui permet d'en déduire tout un ensemble de comportements potentiels, à même de décrire des phénomènes économiques. Le macroéconomiste n'a pas uniquement pour tâche de bâtir un logiciel de politiques économiques mis à disposition des gouvernants, il doit également être capable d'offrir une grille de lecture des événements macroéconomiques. Et une telle grille de lecture permet d'apporter un jugement, plus ou moins informel, sur la réussite potentielle de telle ou telle politique économique. En cela, on peut affirmer que Sargent défend une autre ambition, une ambition supplémentaire, pour la NEC.

\subsection{PRENDRE POSITION DANS LES DEBATS}

Au-delà de son utilité pour interpréter et expliquer des phénomènes économiques passés, la théorie des anticipations rationnelles est donc aussi un outil d'ordre politique, dans le sens où elle entend alimenter les débats autour de l'efficacité des politiques de désinflation au début des années 1980.

Tout d'abord, la théorie des anticipations rationnelles porte en elle une certaine explication de la stagflation. Cette explication est à l'origine de ce que Cristina Romer nomme l'« ideas hypothesis »: "les idées économiques [dans les années 1970] constituent une des causes fondamentales de la Grande Inflation », car « les décideurs des politiques monétaire et budgétaire étaient contraints ou guidés par un paradigme économique erroné »(Romer, 2005, 177)27. Baignés dans la croyance prétendue keynésienne de la stabilité dans le temps de la courbe de Phillips et de la possibilité de maintenir durablement le chômage à un niveau faible, en échange de quelques points

\footnotetext{
27 Dans la même veine, voir également Romer et Romer (2002a ; 2002b ; 2004) et Nelson (2005).
} 
supplémentaires d'inflation, les décideurs ont mis en place tout au long des années 1960 des politiques de stimulation de la demande ${ }^{28}$.

Pour Sargent, le régime keynésien de politique économique a fait évoluer petit à petit les anticipations des agents. Travailleurs et entreprises ont commencé à « anticiper des taux d'inflation élevés et [à s'affronter] dans des négociations à caractère inflationniste à la lumière de ces anticipations ", du fait même que " les politiques monétaires et budgétaires du moment et à venir garantissaient ces anticipations » (Sargent, 2013, 39). Comme on l'a vu ci-dessus, la domination de ces politiques dites keynésiennes pendant vingt ans est une raison qui rendait Sargent dubitatif vis-à-vis de la capacité de l'administration Thatcher à réduire l'inflation en Grande-Bretagne (ibid. 115).

En parallèle de ce diagnostic, la théorie des anticipations rationnelles permet à Sargent de positionner la NEC dans les débats de l'époque sur les remèdes à la stagflation. En suivant les contributions rassemblées dans Rational Expectations and Inflation, on peut considérer que découle de la théorie des anticipations rationnelles une certaine prise de position sur la politique économique à même de résoudre les problèmes de la stagflation, position qui contraste, bien entendu, avec celle défendue plus ou moins unanimement par les keynésiens de premier plan, mais aussi avec les propositions des monétaristes.

La question qui domine les débats est celle du coût en termes de chômage et de perte de production d'une politique de désinflation. Pour Sargent, deux camps s'opposent ici. Le premier est convaincu que :

This inflation has a stubborn, self-sustaining momentum and that either it simply is not susceptible to cure by conventional measures of monetary and fiscal restraint, or, in terms of the consequent widespread and sustained unemployment, the cost of eradicating inflation by monetary and fiscal measures would be prohibitively high. (Ibid. 38)

Pour des keynésiens tels que Tobin, Samuelson ou Okun, le coût en termes de ratio de sacrifice est bien trop élevé, car l'inflation n'évolue que trop lentement du fait de son inertie $^{29}$. Il est alors nécessaire de considérer des mesures autres que des mesures « conventionnelles », telles que, par exemple, une politique des revenus (Okun, 1977).

Le point de vue des anticipations rationnelles, selon Sargent, implique de rejeter cette vision d'un « momentum ». Si l'inflation semble lente à réagir, c'est parce que le régime keynésien de politique économique a fixé les agents dans un certain régime d'anticipations, et toute réévaluation à la baisse de l'inflation anticipée est lente. Mais si

\footnotetext{
${ }^{28}$ Forder (2014) remet en cause cette vision de la macroéconomie des années 1960, qu'il considère comme une reconstruction a posteriori, datant des années 1970.

${ }^{29}$ Les keynésiens de la "vieille » génération ne sont pas les seuls à défendre l'idée d'un ratio de sacrifice élevé. Robert Gordon, entre autres, ne cesse d'insister sur le rôle joué dans les mécanismes de l'inflation par les rigidités et les délais d'ajustement (Goutsmedt et Rubin, 2018). A l'époque où Sargent écrit, une importante littérature sur les rigidités dans un cadre d'anticipations rationnelles, que l'on appellera plus tard «nouvelle économie keynésienne », s'est déjà développée (Fischer, 1977, Phelps et Taylor, 1977). Sargent n'en fait néanmoins pas mention.
} 
cette réévaluation des anticipations est lente, c'est justement parce qu'elle est garantie par le régime actuel de politique économique. Sargent défend une position qui peut être jugée comme plus optimiste, dans le sens où il est selon lui possible que la désinflation soit à la fois rapide et peu coûteuse. Mais pour cela, il faut transformer radicalement le régime de politiques économiques, et que ce changement soit jugé crédible par les agents ${ }^{30}$.

Cet optimisme est tout relatif tant on a vu ci-dessus que les conditions nécessaires à cette crédibilité peuvent être restrictives. Il faut envisager les préconisations politiques de Sargent comme des préconisations de long terme. Au sujet de la coordination entre politique monétaire et politique budgétaire, par exemple, il nous explique :

Superior outcomes would be achieved if the responsibilities of the monetary and fiscal authorities were to be |textit\{legislatively or constitutionally restricted\} so as to determine in advance which institutions are to lead and which are to follow. (Sargent, 2013, 36, je souligne)

On retrouve d'ailleurs ce point dans le seul article vraiment politique écrit par Lucas. Pour ce dernier, il est nécessaire de penser des institutions économiques qui permettent " d'offrir un environnement stable et prévisible pour le secteur privé » (Lucas, 1980b, 210, je souligne). Un nouveau régime de politique économique qui garantisse des anticipations stables prend du temps à s'établir. Mais c'est ce qui justifie de tenter de le mettre en place le plus rapidement possible, pour sortir de l'impasse du régime keynésien.

Cependant, Sargent ne se contente pas de s'opposer aux keynésiens. Il prend également position contre les monétaristes et leur programme de désinflation graduelle. Inspirée par les monétaristes, l'administration Thatcher se proposait de mettre en place, en 1979, un plan d'action sur cinq ans, qui visait à réduire progressivement le taux de croissance de l'offre de monnaie (en ciblant l'agrégat monétaire M3), ainsi que les dépenses publiques (Sargent, 2013, 124-25). Pour Sargent, l'idée monétariste centrale de ce plan est que « même face à des déficits budgétaires persistants, en maniant l'offre de monnaie comme il le faut, le gouvernement peut éviter l'inflation » (ibid. 125-26). Mais un tel plan se heurte à la question de sa crédibilité :

The preceding argument raises questions about the credibility of an announced plan to lower the monetary growth rate and to move to as k-percent, while simultaneously projecting substantial government budget deficits for the several years in the immediate future. The doubtful credibility of such a plan stems from the fact that a large permanent real government deficit is

\footnotetext{
${ }^{30}$ Il semble clair au début des années 1980, que le changement radical de politique monétaire opéré à la Fed, par Paul Volcker, depuis octobre 1979, constituait un test de ce mécanisme exposé par Sargent. Ainsi, à l'époque, Blanchard (1984), Gordon et King (1982), et d'autres ont tenté de montrer empiriquement que la désinflation n'était pas si rapide que ce que pouvaient laisser penser les tenants des anticipations rationnelles et du concept de crédibilité (voir Goutsmedt et al., 2017). Ici encore, Sargent ne discute pas ces résultats
} 
simply incompatible with a k-percent rule for the monetary base. A minimal requirement that a plan be credible is that it be feasible in the first place. (Ibid. 127)

La politique monétaire ne peut être jugée crédible que si les agents considèrent que les déficits budgétaires disparaîtront dans le futur et que des excédents seront générés pour rembourser l'endettement des périodes précédentes. Mais après plusieurs années de politiques keynésiennes, il n'est pas certain que les agents fassent confiance au gouvernement pour réduire les déficits, s'ils soupçonnent que le gouvernement peut être tenté de stopper la réduction du déficit après quelques mois. Autrement dit, pour Sargent, pour que la politique de désinflation soit crédible, il faut qu'elle apparaisse réalisable dès aujourd'hui, et il faut donc que les déficits soient supprimés le plus tôt possible. Sargent préconise ainsi des mesures plus drastiques et immédiates, car le gradualisme laisse ouvert la possibilité que l'engagement initial ne soit pas tenu sur le long terme, et rend donc cette politique non-crédible, et ainsi coûteuse en termes de chômage et de baisse de production.

Ainsi, Sargent est animé par l'idée d'utiliser la théorie des anticipations rationnelles pour interpréter les événements présents et comme outil d'analyse des politiques économiques. Elle constitue un guide pour juger de la potentielle réussite d'une politique. Les modèles de la NEC deviennent alors un outil intellectuel, d'un ordre tout autre que l'outil technique proposé par Lucas.

\section{CONCLUSION}

Si Lucas a été perçu comme "le maître de la méthodologie », c'est sans doute en partie car il affiche une certaine cohérence et une certaine clarté dans ses préconisations. La lecture de ses articles majeurs donne une idée de ce que devrait être, selon lui, la bonne macroéconomie. Pour qu'elle soit rigoureuse, elle doit s'émanciper le plus possible de l'idéologie. Mathématiques et séparation stricte entre le modèle et la réalité sont les instruments de cette émancipation. Nul doute que ces considérations sur la macroéconomie sont en partie des prises de position stratégiques : il s'agit de pousser les keynésiens dans les cordes, en renvoyant dans le camp adverse les accusations de parti pris idéologique.

Mais par la même occasion, à travers la vision idéale de la macroéconomie qu'il transmet, Lucas semble ainsi appauvrir le statut du macroéconomiste, réduit au rôle de technicien dédié à la simulation informatique de politiques économiques. C'est donc à une réextension de l'objet des modèles macroéconomiques que nous convie Sargent au début des années 1980. L'économie a vocation à expliquer les phénomènes réels, à nous éclairer au milieu de la complexité du monde. Les anticipations rationnelles, en tant qu'intuition fondamentale pour comprendre les interactions économiques, nous offrent un point de départ pour penser des phénomènes macroéconomiques et pour expliquer 
les mécanismes sous-jacents. La grille de lecture qui en résulte doit enrichir notre compréhension de l'économie réelle et ainsi favoriser notre capacité à agir sur elle.

Lucas et Sargent sont fondamentalement complémentaires. Si le premier est rapidement considéré comme "le maître de la méthodologie ", c'est sans doute qu'il a su prendre à l'époque ses distances de prises de position plus contestables, en affichant les oripeaux du scientifique détaché de l'idéologie et des considérations trop immédiates. Par sa position, il s'est efforcé de rester en surplomb, tout en servant ainsi d'appui et de force légitimante pour les nouveaux classiques qui n'affichaient pas la même «pureté » méthodologique. Car on voit bien ce que Sargent choisit d'abandonner à travers sa démarche : qu'est-ce qui permet véritablement de juger qu'une politique est, ou va être, crédible (et donc anticipée par les agents) ? A quel moment considère-t-on qu'il y a consensus politique ? Que sait-on véritablement de la conception que les individus peuvent avoir de la politique économique ? Un certain nombre d'éléments de son analyse sont soumis au « jugement » et à la « discrétion » de l'économiste, comme il le reconnaît lui-même. Il renonce en quelque sorte en partie à la volonté de rigueur scientifique de Lucas, pour offrir un atour plus séduisant à la NEC. Mais nul doute que la NEC n'aurait pas connu un tel succès si ces deux ambitions, paradoxalement tout aussi complémentaires qu'antagoniques, n'avaient coexisté31.

Cette démarche de Sargent nous incite à regarder vers une direction pour le moment ignorée par l'historiographie actuelle de la macroéconomie des années 1970 et 1980. Lucas est sans doute le personnage le plus « académique » de la NEC. Il faut s'intéresser à Sargent, ainsi qu'à d'autres macroéconomistes que l'on pourrait rattacher à la NEC (John Kareken, Preston Miller, Neil Wallace), pour constater l'implication et l'implantation du courant de pensée dans les institutions de la politique économique. Dès 1977, un groupe de recherche se construit au sein de la Federal Reserve Bank de Minneapolis, publiant régulièrement dans la Quarterly Review de l'institution. Le président de la banque à l'époque, Mark Willes, se fait le héraut des recherches du groupe au sein du Federal Open Market Committee (McGregor, 2013). Un réseau se construit ainsi pour diffuser les idées de la NEC à une plus large échelle que le champ académique, auquel une lecture trop « lucassienne » de l'histoire de la macroéconomie semble les cantonner.

\section{RÉFÉRENCES}

Arrow, Kenneth J. et Gerard Debreu. 1954. Existence of an Equilibrium for a Competitive Economy. Econometrica, 22 (3) : 265-290.

\footnotetext{
${ }^{31}$ Et Lucas lui-même reconnaît parfaitement l'ambition de Sargent, dans une lettre du 14 octobre 1981, suite à un exposé de ce dernier : "In the case of your paper, I'm not so sure it is devoid of new "results". Theorems aside, the paper does show the way for introducing some content into the application of rational expectations modes to the interpretation of individual events. I had been kind of taking it for granted that this was not a possibility, but I had not seen the potential of a serious use of a broader range of historical evidence to supplement the economic time series » (Lucas Archives, Box 4, Folder : 1981).
} 
Begg, David K. H. 1982. The Rational Expectations Revolution in Macroeconomics. Theories and Evidence. Banbury (UK) : Philip Allan.

Blanchard, Olivier J. J. 1984. The Lucas Critique and the Volcker Deflation. American Economic Review, 74 (2) : 211-215.

Blinder, Alan S. 1979. Economic Policy and the Great Stagflation. London : Academic Press.

Boumans, Marcel. 1997. Lucas and Artificial Worlds. History of Political Economics, 29 (4) : 63_88.

Bruno, Michael et Jeffrey D. Sachs. 1985. Economics of Worldwide Stagflation. Cambridge (MA) : Harvard University Press.

Buiter, Willem H. 1980. The Macroeconomics of Dr. Pangloss. A Critical Survey of the New Classical Macroeconomics. The Economic Journal, 90 (357) : 34-50.

Cagan, Phillip. 1956. The Monetary Dynamics of Hyperinflation. In Milton Friedman (éd.), Studies in the Quantity Theory of Money, Chicago : University of Chicago Press. 25-117.

Cockett, Richard. 1995. Thinking the Unthinkable: Think-tanks and the Economic Counter-Revolution 1931-1983 . New York : Harper Collins.

De Vroey, Michel. 2009. Keynes, Lucas : d'une macroéconomie à l'autre. Paris : Dalloz.

De Vroey, Michel. 2011. Lucas on the Relationship Between Theory and Ideology. Economics : The Open-Access, Open-Assessment E-Journal, 5 (2011-4) : 1-39.

De Vroey, Michel. 2015. A History of Modern Macroeconomics from Keynes to Lucas and Beyond. Cambridge (UK) : Cambridge University Press.

Edwards, Lee. 2013. Leading the Way: The Story of Ed Feulner and the Heritage Foundation. New York : Doubleday Religious Publishing Group.

Fischer, Stanley. 1977. Long-Term Contracts, Rational Expectations, and the Optimal Money Supply Rule. Journal of Political Economy, 85 (1) : 191-205.

Forder, James. 2014. Macroeconomics and the Phillips Curve Myth. Oxford : Oxford University Press. Friedman, Milton. 1953. The Methodology of Positive Economics. In Milton Friedman (éd.), Essays in Positive Economics, Chicago : University of Chicago Press. 3-43.

Gordon, Robert J. et Stephen R. King. 1982. The Output Cost of Disinflation in Traditional and Vector Autoregressive Models. Brookings Papers on Economic Activity, 1982 (1) : 205-244.

Goutsmedt, Aurélien. 2017. Stagflation and the Crossroad in Macroeconomics: The Struggle between Structural and New Classical Macroeconometrics. Documents de travail du Centre d'Economie de la Sorbonne, 2017.43.

Goutsmedt, Aurélien, Erich Pinzon-Fuchs, Matthieu Renault et Francesco Sergi. 2017. Reacting to the Lucas Critique: The Keynesians' Pragmatic Replies. Documents de travail du Centre d'Economie de la Sorbonne, 2017.42.

Goutsmedt, Aurélien et Goulven Rubin. 2018. Robert J. Gordon and the introduction of the Natural Rate Hypothesis in a Keynesian Framework. History of Economic Ideas, Forthcoming 2018.

Hall, Robert E. 1996. Robert Lucas, Recipient of the 1995 Nobel Memorial Prize in Economics. The Scandinavian Journal of Economics, 98 (1) : 33-48.

Hoover, Kevin D. 1988. The New Classical Macroeconomics: A Sceptical Inquiry. Oxford : Basil Blackwell.

Kim, Kyun. 1988. Equilibrium Business Cycle Theory in Historical Perspective. Cambridge (UK) : Cambridge University Press. 
Klamer, Arjo. 1984. The New Classical Macroeconomics. Conversations with the New Classical Economists and Opponents. Brighton (UK) : Wheatsheaf Books.

Krugman, P. 2000. How Complicated does the Model have to be? Oxford Review of Economic Policy, $16(4): 33-42$.

Kydland, Finn E. et Edward C. Prescott. 1982. Time to Build and Aggregate Fluctuations. Econometrica, 50 (6) : 1345-1370.

Lucas, Robert E. 1972. Expectations and the Neutrality of Money. Journal of Economic Theory, 4 (2) : 103-124.

Lucas, Robert E. 1976. Econometric Policy Evaluation: A Critique. Carnegie-Rochester Conference Series on Public Policy, 1 : 19-46.

Lucas, Robert E. 1980a. Methods and Problems in Business Cycle Theory. Journal of Money, Credit and banking, 12 (4) : 696-715.

Lucas, Robert E. 1980b. Rules, Discretion, and the Role of the Economic Advisor. In Stanley Fischer (éd.), Rational Expectations and Economic Policy, Chicago : University of Chicago Press. 199-210.

Lucas, Robert E. 1981. Studies in Business-Cycle Theory. Cambridge, MA : MIT Press.

Lucas, Robert E. et Leonard A. Rapping. 1969. Real Wages, Employment, and Inflation. Journal of Political Economy, 77 (5) : 721-754.

Lucas, Robert E et Leonard A Rapping. 1972. Unemployment in the Great Depression : Is there a full explanation ? Journal of Political Economy, 80 (1) : 186-191.

Lucas, Robert E. et Thomas J. Sargent. 1979. After Keynesian Macroeconomics. Federal Reserve of Minneapolis Quarterly Review, 3 : 1-6.

Mäki, Uskali. 1998. Instrumentalism. In Handbook of Economic Methodology, Edward Elgar Publishing. 253-256.

Mäki, Uskali. 2009a. Reading the Methodological Essay in XX-century Economics. A Map of Multiple Perspectives. In Uskali Mäki (éd.), The Methodology of Positive Economics: Reflections on the Milton Friedman Legacy, Cambridge (UK) : Cambridge University Press. 47-67.

Mäki, Uskali. 2009b. Unrealistic Assumptions and Unnecessary Confusions: Rereading and Rewriting F53 as a Realist Statement. In Uskali Mäki (éd.), The Methodology of Positive Economics: Reflections on the Milton Friedman Legacy, Cambridge (UK) : Cambridge University Press. 90-116.

Mäki, Uskali. 2012. Realism and Antirealism about Economics. In Uskali Mäki, Dov M. Gabbay, Paul Thagard et John Woods (éds.), Philosophy of Economics, Amsterdam : Noth-Holland. 3-24.

McGregor, Rob Roy etWarren Young. 2013. Federal Reserve Bank Presidents as Public Intellectuals. History of Political Economy, 45 : 166-190.

Miller, Preston J. 1994. The Rational Expectations Revolution: Readings from the Front Line. Cambridge (MA) : MIT Press.

Mitchell, Wesley C. 1923. Business Cycles. In Business Cycles and Unemployment, New-York : NBER. 7-20.

Muth, John F. 1961. Rational Expectations and the Theory of Price Movements. Econometrica, 29 (3): 315-335.

Nelson, Edward. 2005. The Great Inflation of the Seventies: What really Happened? Advances in Macroeconomics, 5 (1) : 1534-1613.

Okun, Arthur M. 1977. The Great Stagflation Swamp. Challenge, 20 (5) : 6-13. 
Phelps, Edmund S. et John B. Taylor. 1977. Stabilizing Powers of Monetary Policy under Rational Expectations. Journal of Political Economy, 85 (1) : 163-190.

Reiss, Julian. 2013. Philosophy of Economics: A Contemporary Introduction, New York: Routledge.

Romer, Christina D. 2005. Commentary on Origins of the Great Inflation by Meltzer. Federal Reserve Bank of St. Louis Review, 87 (2) : 177-186.

Romer, Christina D. et David H. Romer. 2002a. A Rehabilitation of Monetary Policy in the 1950's. American Economic Review, 92 (2) : 121-127.

Romer, Christina D. et David H. Romer. 2002b. The Evolution of Economic Understanding and Postwar Stabilization Policy. NBER Working Paper, No 9274.

Romer, Christina D. et David H. Romer. 2004. Choosing the Federal Reserve Chair: Lessons from History. The Journal of Economic Perspectives, 18 (1) : 129-162.

Sargent, Thomas J. 1980. Rational Expectations and the Reconstruction of Macroeconomics. Federal Reserve Bank of Minneapolis Quarterly Review, 4 (3) : 15-19.

Sargent, Thomas J. 1982. The Ends of Four Big Inflations. In Robert Ernest Hall (éd.), Inflation: Causes and Effects, Chicago : University of Chicago Press. 41-98.

Sargent, Thomas J. 1983. Stopping Moderate Inflations: The Methods of Poincare and Thatcher. In Rudiger Dornbusch et Mario Henrique Simonsen (éds.), Inflation, Debt and Indexation, Cambridge (MA) : MIT Press. 54-96.

Sargent, Thomas J. 1985. Reaganomics and Credibility. In Albert Ando, Nihon Ginko, Roger E. A. Farmer et Yoshio Suzuki (éds.), Monetary Policy in Our Times, Cambridge (MA) : MIT Press. 19-35.

Sargent, Thomas J. 2012. Nobel Lecture: United States Then, Europe Now. Journal of Political Economy, 120 (1) : 1-40.

Sargent, Thomas J. 2013. Rational Expectations and Inflation. Princeton : Princeton University Press.

Sargent, Thomas J. et Neil Wallace. 1976. Rational Expectations and the Theory of Economic Policy. Journal of Monetary Economics, 2 (2) : 169-183.

Sent, Esther-Mirjam. 1998. The Evolving Rationality of Rational Expectations: An Assessment of Thomas Sargent's Achievements. New York : Cambridge University Press.

Sergi, Francesco. 2017. De la révolution lucasienne aux modèles DSGE. Réflexions sur la modélisation macroéconomique contemporaine. Université Paris I Panthéon-Sorbonne : Thèse de doctorat.

Smith, James A. 1993. Idea Brokers: Think Tanks And The Rise Of The New Policy Elite . New York: Simon and Schuster.

Snowdon, Brian et Howard R. Vane. 2005. Modern Macroeconomics: Its Origins, Development and Current State. Cheltenham : Edward Elgar Publishing.

Solow, Robert. 1978. Summary and Evaluation. In After the Phillips Curve: Persistence of High Inflation and High Unemployment, Federal Reserve of Boston Conference Series, Federal Reserve of Boston. 203-209.

Solow, Robert. 1980. What to Do (Macroeconomically) when OPEC Comes. In Rational Expectations and Economic Policy, A Conference Report - National Bureau of Economic Research, Chicago : University of Chicago Press. 249-268.

Stein, Jerome L. 1981. Monetarist, Keynesian, and new classical economics. The American Economic Review, 71 (2) : 139-144.

Tobin, James. 1980. Are New Classical Models Plausible Enough to Guide Policy? Journal of Money, Credit and Banking, 12 (4) : 788-799. 
Tobin, James. 1981. The Monetarist Counter-Revolution Today. An Appraisal. The Economic Journal, 91 (361) : 29-42.

Vercelli, Alessandro. 1991. Methodological Foundations of Macroeconomics: Keynes and Lucas. Cambrige (UK) : Cambridge University Press.

Willes, Mark H. 1978. Point of View: Surprises Can Be Bad Economic Policy. New York Times : F28.

Woodford, Michael. 2003. Interest and Prices. Foundations of a Theory of Monetary Policy. Princeton: Princeton University Press. 